\title{
Radiative quark jet function with an external gluon
}

\author{
Ze Long Liu, ${ }^{a}$ Matthias Neubert, ${ }^{b, c}$ Marvin Schnubel ${ }^{b}$ and Xing Wang ${ }^{b}$ \\ ${ }^{a}$ Institut für Theoretische Physik \& Albert Einstein Center, Universität Bern, \\ Sidlerstrasse 5, CH-3012 Bern, Switzerland \\ ${ }^{b}$ PRISMA+ Cluster of Excellence \& Mainz Institute for Theoretical Physics, \\ Johannes Gutenberg University, \\ Staudingerweg 9, 55128 Mainz, Germany \\ ${ }^{c}$ Department of Physics \& LEPP, Cornell University, \\ Ithaca, NY 14853, U.S.A. \\ E-mail: zelongliu@itp.unibe.ch, neubertm@uni-mainz.de, \\ maschnub@uni-mainz.de, x.wang@uni-mainz.de
}

ABSTRACT: Factorization theorems in soft-collinear effective theory at subleading order in power counting involve "radiative jet functions", defined in terms of matrix elements of hard-collinear fields with a soft momentum emitted from inside the jet. Of particular importance are the radiative quark jet functions with an external photon or gluon, which arise e.g. in the factorization theorems for the Higgs-boson amplitudes $h \rightarrow \gamma \gamma, h \rightarrow g g$ and $g g \rightarrow h$ induced by light-quark loops. While the photon case has been studied extensively in previous work, we present here a detailed study of the radiative jet function with an external gluon. We calculate this jet function at one- and two-loop order, derive its one-loop anomalous dimension and study its renormalization-group evolution.

KEYwords: Effective Field Theories, Perturbative QCD, Renormalization Group, Resummation

ArXiv ePrint: 2112.00018 


\section{Contents}

1 Introduction 1

2 Two-loop calculation of the bare jet function 3

$\begin{array}{lll}3 & \text { Renormalization of the jet function } & 6\end{array}$

4 RG evolution of the jet function $\quad 10$

5 Conclusions $\quad 12$

$\begin{array}{ll}\text { A Master integrals } & 12\end{array}$

B Bare two-loop coefficients to $\mathcal{O}\left(\epsilon^{2}\right) \quad 14$

\section{Introduction}

Soft-collinear effective theory (SCET) is a helpful tool to study high-energy cross sections and decay rates sensitive to several hierarchical mass scales (see e.g. [1] and references therein). Deriving factorization theorems, consisting of functions each depending on a single scale, is an important step towards understanding the dynamics of such objects. Typically, the various functions receive contributions from different momentum regions in Feynman diagrams. The hard functions are the Wilson coefficients obtained by matching the Standard Model onto SCET. Jet and soft functions, on the other hand, are defined in terms of matrix elements of hard-collinear and soft fields in the effective theory, respectively (sometimes referred to as "collinear" and "ultra-soft"). In particular, the jet functions consist of non-local products of hard-collinear fields dressed by Wilson lines, and they often depend on an intermediate short-distance scale between the hard-scattering scale of the process and the soft scale.

In the past years, there has been a growing interest in understanding factorization at subleading power in scale ratios. Beyond the leading power a large variety of hard, hardcollinear and soft functions appear. In particular, while at leading power soft emissions are eikonal and can be described by soft Wilson lines, at subleading power the emission of soft quarks needs to be taken into account. At subleading order in the SCET expansion, there is a unique interaction that couples a soft quark to hard-collinear quarks and gauge fields. In the notation of [2] it reads

$$
\mathcal{L}_{q \xi_{n}}^{(1 / 2)}(x)=\bar{q}_{s}\left(x_{-}\right) W_{n}^{\dagger}(x) i \not D_{n}^{\perp} \xi_{n}(x)+\text { h.c. },
$$

where $\xi_{n}$ is a hard-collinear quark spinor subject to the constraint $\not h \xi_{n}=0$. We introduce null-vectors $n^{\mu}=(1, \mathbf{n})$ aligned with the jet direction, and $\bar{n}^{\mu}=(1,-\mathbf{n})$ pointing in the 
opposite direction, such that $n \cdot \bar{n}=2$. The quantity $W_{n}$ denotes a hard-collinear Wilson line (a path-ordered exponential of a line integral over the hard-collinear gluon field $\bar{n} \cdot A_{n}$ ), $D_{n}^{\mu}$ is a covariant hard-collinear derivative, and $q_{s}$ is a soft quark field. The hard-collinear particles carry large momentum along the direction $n^{\mu}$, whereas the momenta of soft fields are small. As a consequence, in interactions with hard-collinear fields a soft quark field must be multipole expanded [2], and we denote $x_{-}^{\mu}=(\bar{n} \cdot x) \frac{n^{\mu}}{2}$ in the above relation.

Scale factorization at subleading power in SCET turns out to be an unexpectedly rich and complicated problem due to the presence of endpoint divergences in convolution integrals [3-9]. Only recently, the derivation of a renormalized factorization theorem for a subleading-power quantity, in which endpoint divergences are regularized using plus-type subtractions, has been accomplished for the first time $[8,10,11]$. The pseudo-observable considered in these works was the Higgs-boson decay $h \rightarrow \gamma \gamma$ induced via a $b$-quark loop. In the factorization formula, the radiative quark jet function with an external collinear photon arose [12], which is an object well known from earlier studies of QCD factorization in the radiative $B$-meson decay $B^{-} \rightarrow \gamma \ell^{-} \bar{\nu}[13,14]$. The term "radiative jet function" indicates that, in contrast to the jet functions appearing in leading-power SCET problems, a soft quark is emitted from inside the jet $[3,15-17]$.

Generalizing the $h \rightarrow \gamma \gamma$ decay to its non-abelian cousins, i.e. the decay $h \rightarrow g g$ or the production process $g g \rightarrow h$, one encounters the radiative quark jet function $J_{g}\left(p^{2}\right)$ with an external collinear gluon [18], which is the main subject of this work. The external gluon has a virtuality much lower than the short-distance hard-collinear scale, and hence it must be described by a collinear rather than a hard-collinear field. In fact, in the $p p \rightarrow h$ production process via gluon-gluon fusion, the proton matrix element of two collinear gluons fields defines the gluon distribution function of the proton. Generalizing the treatment of the radiative jet function with an external photon in [12], we thus define the function $J_{g}\left(p^{2}\right)$ via the matching relation

$$
\int d^{d} x e^{i p_{s} \cdot x_{-}} T\left(W_{n}^{\dagger} i \not D_{n}^{\perp} \xi_{n}\right)(x)\left(\bar{\xi}_{n} W_{n}\right)(0)=J_{g}\left(\bar{n} \cdot \mathcal{P} n \cdot p_{s}\right) \mathcal{A}_{c, n}^{\perp}(0) \frac{i \not h}{2 n \cdot p_{s}+i 0},
$$

where $\mathcal{A}_{c, n}^{\perp \mu}$ denotes the collinear gauge-invariant gluon field defined by [1, 19]

$$
\mathcal{A}_{c, n}^{\perp \mu}=W_{c, n}^{\dagger}\left(i D_{c, n}^{\perp \mu} W_{c, n}\right)
$$

and $\bar{n} \cdot \mathcal{P}=-i \bar{n} \cdot \partial$ is a differential operator projecting out the large component of the collinear momentum carried by the gluon field. Moreover, $p_{s}$ denotes the momentum carried away by the multipole-expanded soft quark field in (1.1), and since $p_{s} \cdot x_{-}=p_{s+} \cdot x=$ $\frac{1}{2} n \cdot p_{s} \bar{n} \cdot x$, the matching coefficient $J_{g}$ can only depend on $n \cdot p_{s}$. Note that the right-hand side of (1.2) is invariant under longitudinal Lorentz boosts, under which $n^{\mu} \rightarrow e^{-\eta} n^{\mu}$ and $\bar{n}^{\mu} \rightarrow e^{\eta} \bar{n}^{\mu}$ (type-III reparameterization invariance [20]).

The matching coefficient $J_{g}\left(p^{2}\right)$ is, by construction, insensitive to long-distance physics, since it accounts for the effects of integrating out hard-collinear modes. The simplest way to calculate this coefficient is to evaluate both sides of the matching relation between the 
vacuum and an on-shell gluon state (with virtuality $k^{2}=0$ ). This yields

$$
\int d^{d} x e^{i p_{s} \cdot x_{-}}\left\langle g(k, a)\left|T\left(W_{n}^{\dagger} i \not D_{n}^{\perp} \xi_{n}\right)(x)\left(\bar{\xi}_{n} W_{n}\right)(0)\right| 0\right\rangle=g_{s} t_{a} \ddagger_{\perp}^{*}(k) \frac{i \not h}{2 n \cdot p_{s}+i 0} J_{g}\left(p^{2}\right),
$$

where $g_{s}$ is the strong coupling, and $k, \varepsilon$ and $a$ are the momentum, polarization vector and color index of the external gluon, respectively. Because of the conditions $k^{2}=0$ and $p_{s+}^{2}=0$, the jet function $J_{g}\left(p^{2}\right)$ depends on the single non-trivial kinematic invariant

$$
p^{2} \equiv\left(k+p_{s+}\right)^{2}=\bar{n} \cdot k n \cdot p_{s},
$$

in accordance with (1.2). The function $J_{g}\left(p^{2}\right)$ plays a central role in deriving exact $d$ dimensional refactorization conditions, which are at the heart of our method for removing endpoint divergences by means of plus-type subtractions $[8,10,11]$. The study of this radiative jet function thus offers a new window to investigate the structure of powersuppressed corrections to processes with external collinear gluons.

\section{Two-loop calculation of the bare jet function}

Because the jet function depends only on a single, hard-collinear scale $p^{2}$, we can exploit the fact that SCET in a single hard-collinear sector is equivalent to full QCD [2] to replace the hard-collinear quark fields in (1.4) in terms of ordinary QCD fields using $\xi_{n}=\frac{1}{4} \not \hbar \hbar \psi$. We write the result for the bare jet function in $d=4-2 \epsilon$ spacetime dimensions in the general form

$$
J_{g}^{(0)}\left(p^{2}\right)=1+\frac{\alpha_{s, 0}}{4 \pi}\left(-p^{2}-i 0\right)^{-\epsilon} J_{g, 1}^{(0)}+\left(\frac{\alpha_{s, 0}}{4 \pi}\right)^{2}\left(-p^{2}-i 0\right)^{-2 \epsilon} J_{g, 2}^{(0)}+\mathcal{O}\left(\alpha_{s}^{3}\right) .
$$

In our calculations we adopt the background field method for the external gluon [21-25]. It is most convenient to perform the calculation of the expansion coefficients $J_{g, 1}^{(0)}$ and $J_{g, 2}^{(0)}$ in light-cone gauge $\bar{n} \cdot A_{n}=0$, such that the hard-collinear Wilson lines $W_{n}=1$ are trivial. The smaller number of Feynman diagrams and the absence of ghost contributions allow for an efficient computation in this gauge. The free gluon propagator with momentum $l$ in light-cone gauge is given by

$$
\frac{i}{l^{2}+i 0}\left(-g^{\mu \nu}+\frac{\bar{n}^{\mu} l^{\nu}+\bar{n}^{\nu} l^{\mu}}{\bar{n} \cdot l}\right)
$$

where we do not adopt the Mandelstam-Leibbrandt prescription to regularize the singularity at $\bar{n} \cdot l=0$ (see [26] for more details). As a cross check of our results, we have also performed the calculation in a general covariant gauge, finding identical results.

The diagrams contributing at one-loop order to the jet function are shown in figure 1 . Evaluating these graphs we obtain

$$
J_{g, 1}^{(0)}=\left(C_{F}-C_{A}\right) e^{\epsilon \gamma_{E}} \frac{\Gamma(1+\epsilon) \Gamma^{2}(-\epsilon)}{\Gamma(2-2 \epsilon)}\left(2-4 \epsilon-\epsilon^{2}\right),
$$

where $C_{F}=\left(N_{c}^{2}-1\right) /\left(2 N_{c}\right)$ and $C_{A}=N_{c}$ are the eigenvalues of the quadratic Casimir operators in the fundamental and adjoint representations of $\mathrm{SU}\left(N_{c}\right)$. Remarkably, to all 

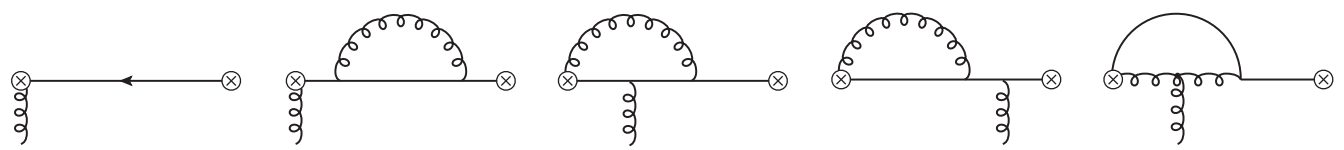

Figure 1. Tree-level and one-loop diagrams contributing to the jet function in light-cone gauge. The left cross corresponds to the spacetime point $x$, where the soft momentum $p_{s}$ flows out, see (1.4). The right cross corresponds to the point 0 . The fourth diagram is scaleless and evaluates to zero.
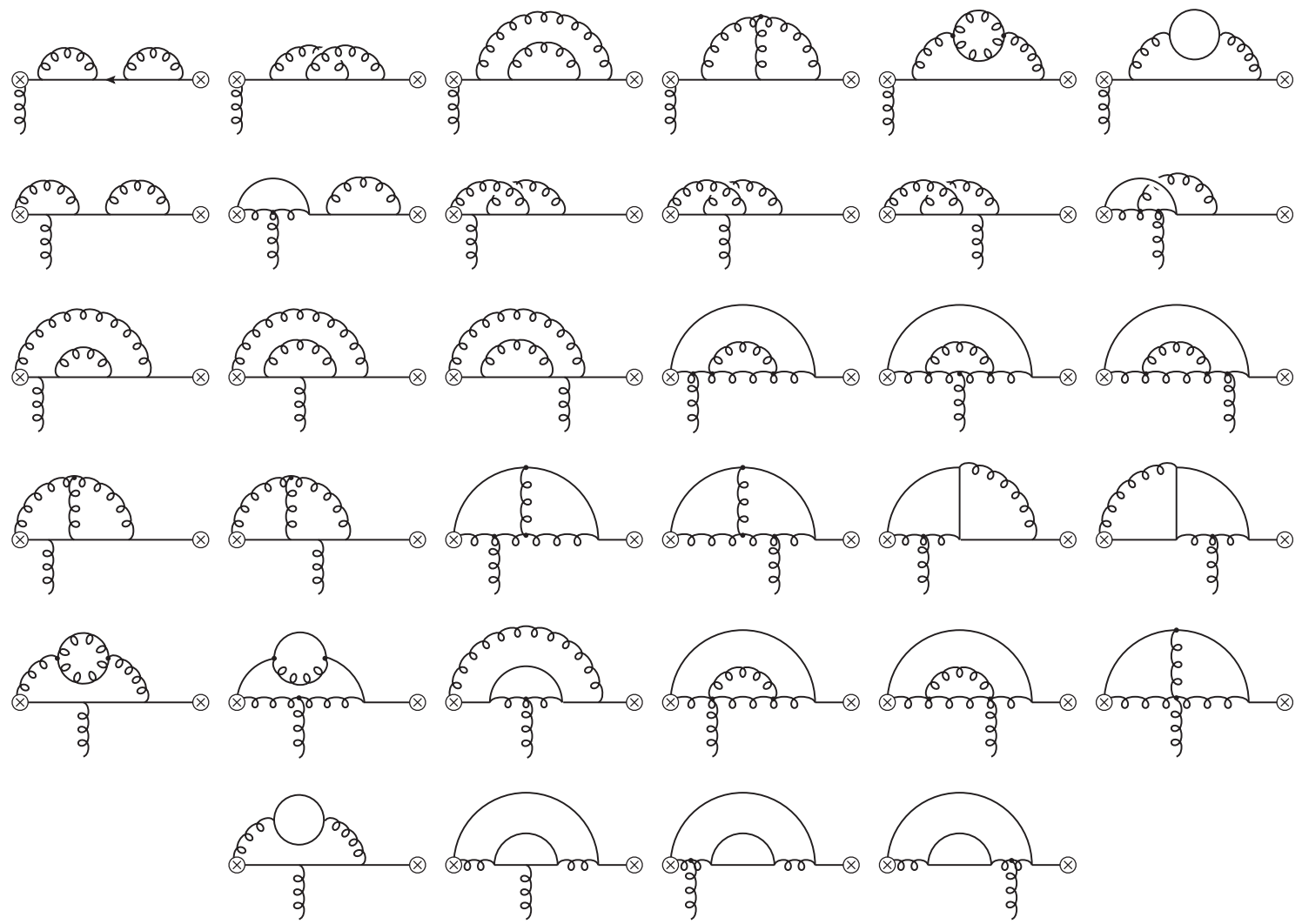

Figure 2. Two-loop diagrams contributing to the jet function in light-cone gauge.

orders in $\epsilon$ this result differs from the corresponding expression for the jet function with an external photon only in the color factor, which is $C_{F}$ in the photon case. We will see below that such a simple relation no longer holds beyond one-loop order.

At two-loop order, the diagrams contributing in light-cone gauge are presented in figure 2. Using simplifications of the Dirac and Lorentz structures, each diagram can be expressed as a linear combination of scalar two-loop integrals of the form

$$
\int d^{d} l_{1} \int d^{d} l_{2} \frac{1}{\prod_{i=1}^{15} \mathcal{D}_{i}^{a_{i}}}
$$


where the propagators are (omitting the $-i 0$ prescription)

$$
\begin{array}{rlrl}
\mathcal{D}_{1}=-l_{1}^{2}, & \mathcal{D}_{2}=-l_{2}^{2}, & \mathcal{D}_{3}=-\left(l_{1}+l_{2}\right)^{2}, \\
\mathcal{D}_{4}=-\left(l_{1}+p\right)^{2}, & \mathcal{D}_{5}=-\left(l_{2}+p\right)^{2}, & & \mathcal{D}_{6}=-\left(l_{1}+l_{2}+p\right)^{2}, \\
\mathcal{D}_{7}=-\left(l_{1}+p-k\right)^{2}, & \mathcal{D}_{8}=-\left(l_{2}+p-k\right)^{2}, & & \mathcal{D}_{9}=-\left(l_{1}+l_{2}+p-k\right)^{2}, \\
\mathcal{D}_{10}=-\bar{n} \cdot l_{1}, & \mathcal{D}_{11}=-\bar{n} \cdot l_{2}, & & \mathcal{D}_{12}=-\bar{n} \cdot\left(l_{1}+l_{2}\right), \\
\mathcal{D}_{13}=-\bar{n} \cdot\left(l_{1}+p\right), & \mathcal{D}_{14}=-\bar{n} \cdot\left(l_{2}+p\right), & \mathcal{D}_{15}=-\bar{n} \cdot\left(l_{1}+l_{2}+p\right) .
\end{array}
$$

After performing partial-fraction decompositions, all scalar Feynman integrals are mapped onto linear complete integral families. We then use the integration-by-parts method as implemented in LiteRed [27], FIRE6 [28] and Kira [29] to reduce these Feynman integrals to linear combinations of ten master integrals, which are listed in appendix A. Using algebraic relations, we have been able to express the master integrals in terms of integrals encountered in the calculation of the jet function with an external photon [12]. In this way, we obtain

$$
J_{g, 2}^{(0)}=C_{F}^{2} K_{F F}+C_{F} C_{A} K_{F A}+C_{A}^{2} K_{A A}+C_{F} T_{F} n_{f} K_{F n_{f}}+C_{A} T_{F} n_{f} K_{A n_{f}},
$$

with

$$
\begin{aligned}
K_{F F}= & \frac{2}{\epsilon^{4}}-\frac{1}{\epsilon^{2}}\left(2+\frac{\pi^{2}}{3}\right)-\frac{1}{\epsilon}\left(4+\frac{\pi^{2}}{2}+\frac{46 \zeta_{3}}{3}\right)-\frac{13}{2}-\frac{\pi^{2}}{6}-39 \zeta_{3}+\frac{\pi^{4}}{5}+\mathcal{O}(\epsilon), \\
K_{F A}= & -\frac{4}{\epsilon^{4}}+\frac{11}{6 \epsilon^{3}}+\frac{1}{\epsilon^{2}}\left(\frac{139}{18}+\frac{\pi^{2}}{2}\right)+\frac{1}{\epsilon}\left(\frac{319}{27}-\frac{\pi^{2}}{18}+\frac{80 \zeta_{3}}{3}\right) \\
& +\frac{1087}{162}-\frac{83 \pi^{2}}{54}+\frac{485 \zeta_{3}}{18}-\frac{49 \pi^{4}}{360}+\mathcal{O}(\epsilon), \\
K_{A A}= & \frac{2}{\epsilon^{4}}-\frac{11}{6 \epsilon^{3}}-\frac{1}{\epsilon^{2}}\left(\frac{103}{18}+\frac{\pi^{2}}{6}\right)-\frac{1}{\epsilon}\left(\frac{413}{54}-\frac{11 \pi^{2}}{18}+\frac{34 \zeta_{3}}{3}\right) \\
& +\frac{100}{81}+\frac{47 \pi^{2}}{27}+\frac{259 \zeta_{3}}{18}-\frac{23 \pi^{4}}{360}+\mathcal{O}(\epsilon), \\
K_{F n_{f}}= & -\frac{2}{3 \epsilon^{3}}-\frac{10}{9 \epsilon^{2}}-\frac{1}{\epsilon}\left(\frac{20}{27}-\frac{\pi^{2}}{9}\right)+\frac{230}{81}+\frac{5 \pi^{2}}{27}+\frac{64 \zeta_{3}}{9}+\mathcal{O}(\epsilon) \\
K_{A n_{f}}= & \frac{2}{3 \epsilon^{3}}+\frac{10}{9 \epsilon^{2}}+\frac{1}{\epsilon}\left(\frac{11}{27}-\frac{2 \pi^{2}}{9}\right)-\frac{491}{81}-\frac{10 \pi^{2}}{27}-\frac{106 \zeta_{3}}{9}+\mathcal{O}(\epsilon)
\end{aligned}
$$

where $T_{F}=\frac{1}{2}$, and $n_{f}$ denotes the number of active quark flavors. The coefficients $K_{F F}$ and $K_{F n_{f}}$ are identical to the corresponding coefficients in the photon case derived in [12], but for the remaining coefficients no simple relation between the two jet functions can be found past one-loop order. In appendix B we give the expansions of these coefficients to second order in the dimensional regulator $\epsilon$. These results would be needed to obtain the renormalized jet function at three-loop order. 


\section{Renormalization of the jet function}

In the $\overline{\mathrm{MS}}$ subtraction scheme, the radiative jet function $J_{g}\left(p^{2}\right)$ can be renormalized multiplicatively in the convolution sense. We write

$$
J_{g}\left(p^{2}, \mu\right)=\int_{0}^{\infty} d x Z_{J_{g}}\left(p^{2}, x p^{2} ; \mu\right) J_{g}^{(0)}\left(x p^{2}\right) .
$$

Note that jet functions at different values of $p^{2}$ mix under renormalization. For the analogous case of the radiative jet function with an external photon studied in [12], this "nonlocal" renormalization condition was derived using the fact that this jet function appears in the QCD factorization theorem for the $B^{-} \rightarrow \gamma \ell^{-} \bar{\nu}$ decay amplitude [30] along with the leading-order light-cone distribution amplitude of the $B$ meson [31], whose renormalization properties have been derived long ago in [32]. In the present case, we can derive the oneloop expression for the renormalization factor $Z_{J_{g}}$ by exploiting the fact that the function $J_{g}\left(p^{2}\right)$ enters the factorization theorem for the Higgs-boson production amplitude in gluongluon fusion, $g g \rightarrow h$, which is structurally very similar to the factorization theorem for the $h \rightarrow \gamma \gamma$ decay amplitude derived in $[8,10,11]$. Using the renormalization-group (RG) equations for the relevant hard and soft functions entering this factorization theorem, ${ }^{1}$ we find that the renormalized jet function in (3.1) obeys the RG evolution equation [18]

$$
\frac{d}{d \ln \mu} J_{g}\left(p^{2}, \mu\right)=-\int_{0}^{\infty} d x \gamma_{J_{g}}\left(p^{2}, x p^{2} ; \mu\right) J_{g}\left(x p^{2}, \mu\right),
$$

where at one-loop order the anomalous dimension is given by

$$
\gamma_{J_{g}}\left(p^{2}, x p^{2} ; \mu\right)=\frac{\alpha_{s}}{\pi}\left[\left(C_{F}-C_{A}\right) \ln \frac{-p^{2}-i 0}{\mu^{2}} \delta(1-x)+\left(C_{F}-\frac{C_{A}}{2}\right) \Gamma(1, x)\right]+\mathcal{O}\left(\alpha_{s}^{2}\right) .
$$

Here $\alpha_{s} \equiv \alpha_{s}(\mu)$ denotes the renormalized (running) QCD coupling, and

$$
\Gamma(y, x)=\left[\frac{\theta(y-x)}{y(y-x)}+\frac{\theta(x-y)}{x(x-y)}\right]_{+}
$$

is the symmetric Lange-Neubert kernel [32]. The plus distribution is defined such that, when $\Gamma(y, x)$ is integrated with a function $f(x)$, one has to replace $f(x) \rightarrow f(x)-f(y)$ under the integral. To all orders in perturbation theory, the coefficient of the scale-dependent logarithm in the anomalous dimension is the difference of the light-like cusp anomalous dimensions in the fundamental and the adjoint representation,

$$
\Gamma_{F}^{\text {cusp }}\left(\alpha_{s}\right)-\Gamma_{A}^{\text {cusp }}\left(\alpha_{s}\right)=\left(C_{F}-C_{A}\right) \frac{\alpha_{s}}{\pi}+\mathcal{O}\left(\alpha_{s}^{2}\right) .
$$

In the limit $C_{A} \rightarrow 0$, the anomalous dimension in (3.3) reduces to the anomalous dimension of the radiative jet function with an external photon. We have also derived the anomalous dimension (3.3) in an independent way using a method recently developed in [34] (and

\footnotetext{
${ }^{1}$ The relevant soft function can be obtained from the soft function in the $h \rightarrow \gamma \gamma$ process [33] by an appropriate replacement of color factors in each Feynman diagram.
} 
applied in [35] to the case of the jet function with an external photon). The result we obtain is in agreement with the expression above. Details of this calculation will be presented elsewhere. Using a general relation between an anomalous dimension and the corresponding $Z$-factor derived in [36], we find that

$$
\begin{aligned}
Z_{J_{g}}\left(p^{2}, x p^{2} ; \mu\right)= & {\left[1+\left(C_{F}-C_{A}\right) \frac{\alpha_{s}}{2 \pi}\left(-\frac{1}{\epsilon^{2}}+\frac{1}{\epsilon} \ln \frac{-p^{2}-i 0}{\mu^{2}}\right)\right] \delta(1-x) } \\
& +\left(C_{F}-\frac{C_{A}}{2}\right) \frac{\alpha_{s}}{2 \pi \epsilon} \Gamma(1, x)+\mathcal{O}\left(\alpha_{s}^{2}\right) .
\end{aligned}
$$

The two-loop coefficient in this expression is currently still unknown.

We now proceed to derive the two-loop expression for the renormalized jet function $J_{g}\left(p^{2}, \mu\right)$. To this end, we first eliminate the bare QCD coupling $\alpha_{s, 0}$ in (2.1) in favor of the renormalized coupling. This yields, again with $\alpha_{s} \equiv \alpha_{s}(\mu)$,

$$
J_{g}^{(0)}\left(p^{2}\right)=1+\frac{\alpha_{s}}{4 \pi} e^{-\epsilon L_{p}} J_{g, 1}^{(0)}+\left(\frac{\alpha_{s}}{4 \pi}\right)^{2}\left[e^{-2 \epsilon L_{p}} J_{g, 2}^{(0)}-\frac{\beta_{0}}{\epsilon} e^{-\epsilon L_{p}} J_{g, 1}^{(0)}\right]+\mathcal{O}\left(\alpha_{s}^{3}\right),
$$

where $L_{p}=\ln \left[\left(-p^{2}-i 0\right) / \mu^{2}\right]$. The second term inside the bracket generates an extra contribution at two-loop order proportional to $\left(C_{F}-C_{A}\right) \beta_{0}$, which contains $1 / \epsilon^{n}$ poles as well as terms that remain finite in the limit $\epsilon \rightarrow 0$. We then renormalize the bare jet function (now expressed in terms of the renormalized coupling) using relation (3.1). At two-loop order this yields

$$
\begin{aligned}
J_{g}\left(p^{2}, \mu\right)= & J_{g}^{(0)}\left(p^{2}\right)+\frac{\alpha_{s}}{4 \pi} \int_{0}^{\infty} d x Z_{J_{g}, 1}\left(p^{2}, x p^{2} ; \mu\right) \\
& +\left(\frac{\alpha_{s}}{4 \pi}\right)^{2}\left[\int_{0}^{\infty} d x Z_{J_{g}, 1}\left(p^{2}, x p^{2} ; \mu\right) e^{-\epsilon L_{p}} J_{g, 1}^{(0)} x^{-\epsilon}+\int_{0}^{\infty} d x Z_{J_{g}, 2}\left(p^{2}, x p^{2} ; \mu\right)\right]+\mathcal{O}\left(\alpha_{s}^{3}\right),
\end{aligned}
$$

where $Z_{J_{g}, n}$ denotes the term in the $Z$-factor multiplying $\left(\frac{\alpha_{s}}{4 \pi}\right)^{n}$. Note that the term in the renormalization factor (3.6) involving the Lange-Neubert kernel gives a non-zero contribution only at two-loop order (first integral in the second line), where it acts on the function $x^{-\epsilon}$. The integral over the two-loop renormalization factor (integral in the third line) is unknown, but in the $\overline{\mathrm{MS}}$ subtraction scheme this integral contains $1 / \epsilon^{n}$ pole terms only. We can thus derive its value from the condition that the expression for the renormalized jet function be finite. This condition yields

$$
\int_{0}^{\infty} d x Z_{J_{g}, 2}\left(p^{2}, x p^{2} ; \mu\right)=C_{F}^{2} k_{F F}+C_{F} C_{A} k_{F A}+C_{A}^{2} k_{A A}+C_{F} T_{F} n_{f} k_{F n_{f}}+C_{A} T_{F} n_{f} k_{A n_{f}},
$$


with

$$
\begin{aligned}
k_{F F}= & \frac{2}{\epsilon^{4}}-\frac{4 L_{p}}{\epsilon^{3}}+\frac{2 L_{p}^{2}}{\epsilon^{2}}+\frac{1}{\epsilon}\left(\frac{\pi^{2}}{2}-2 \zeta_{3}\right), \\
k_{F A}= & -\frac{4}{\epsilon^{4}}+\frac{1}{\epsilon^{3}}\left(8 L_{p}+\frac{11}{2}\right)+\frac{1}{\epsilon^{2}}\left(-4 L_{p}^{2}-\frac{11 L_{p}}{3}-\frac{67}{18}+\frac{\pi^{2}}{6}\right) \\
& +\frac{1}{\epsilon}\left[\left(\frac{67}{9}-\frac{\pi^{2}}{3}\right) L_{p}-\frac{202}{27}-\frac{5 \pi^{2}}{9}+4 \zeta_{3}\right], \\
k_{A A}= & \frac{2}{\epsilon^{4}}-\frac{1}{\epsilon^{3}}\left(4 L_{p}+\frac{11}{2}\right)+\frac{1}{\epsilon^{2}}\left(2 L_{p}^{2}+\frac{11 L_{p}}{3}+\frac{67}{18}-\frac{\pi^{2}}{6}\right) \\
& +\frac{1}{\epsilon}\left[-\left(\frac{67}{9}-\frac{\pi^{2}}{3}\right) L_{p}+\frac{395}{54}-2 \zeta_{3}\right], \\
k_{F n_{f}}= & -\frac{2}{\epsilon^{3}}+\frac{2}{\epsilon^{2}}\left(\frac{4 L_{p}}{3}+\frac{10}{9}\right)+\frac{1}{\epsilon}\left(-\frac{20 L_{p}}{9}+\frac{56}{27}+\frac{\pi^{2}}{9}\right), \\
k_{A n_{f}}= & \frac{2}{\epsilon^{3}}-\frac{2}{\epsilon^{2}}\left(\frac{4 L_{p}}{3}+\frac{10}{9}\right)+\frac{1}{\epsilon}\left(\frac{20 L_{p}}{9}-\frac{47}{27}\right) .
\end{aligned}
$$

The terms multiplying powers of $L_{p}$ in this expression are governed by the cusp anomalous dimension, such that

$$
\int_{0}^{\infty} d x Z_{J_{g}, 2}\left(p^{2}, x p^{2} ; \mu\right)=L_{p}^{2} \frac{\left(\Delta \Gamma_{0}\right)^{2}}{8 \epsilon^{2}}+L_{p}\left[-\frac{\left(\Delta \Gamma_{0}\right)^{2}}{4 \epsilon^{3}}-\frac{\beta_{0} \Delta \Gamma_{0}}{4 \epsilon^{2}}+\frac{\Delta \Gamma_{1}}{4 \epsilon}\right]+\ldots
$$

where $\Delta \Gamma_{0,1}$ are the one- and two-loop coefficients of the difference in (3.5), i.e. [37, 38]

$$
\Delta \Gamma_{0}=4\left(C_{F}-C_{A}\right), \quad \frac{\Delta \Gamma_{1}}{\Delta \Gamma_{0}}=\left(\frac{67}{9}-\frac{\pi^{2}}{3}\right) C_{A}-\frac{20}{9} T_{F} n_{f}
$$

After all $1 / \epsilon^{n}$ poles have been subtracted, we take the limit $\epsilon \rightarrow 0$ to obtain the two-loop expression for the renormalized jet function. The result is

$$
\begin{aligned}
J_{g}\left(p^{2}, \mu\right)= & +\frac{\alpha_{s}}{4 \pi}\left(C_{F}-C_{A}\right)\left(L_{p}^{2}-1-\frac{\pi^{2}}{6}\right) \\
& +\left(\frac{\alpha_{s}}{4 \pi}\right)^{2}\left[C_{F}^{2} \bar{K}_{F F}+C_{F} C_{A} \bar{K}_{F A}+C_{A}^{2} \bar{K}_{A A}+C_{F} T_{F} n_{f} \bar{K}_{F n_{f}}+C_{A} T_{F} n_{f} \bar{K}_{A n_{f}}\right] \\
& +\mathcal{O}\left(\alpha_{s}^{3}\right),
\end{aligned}
$$



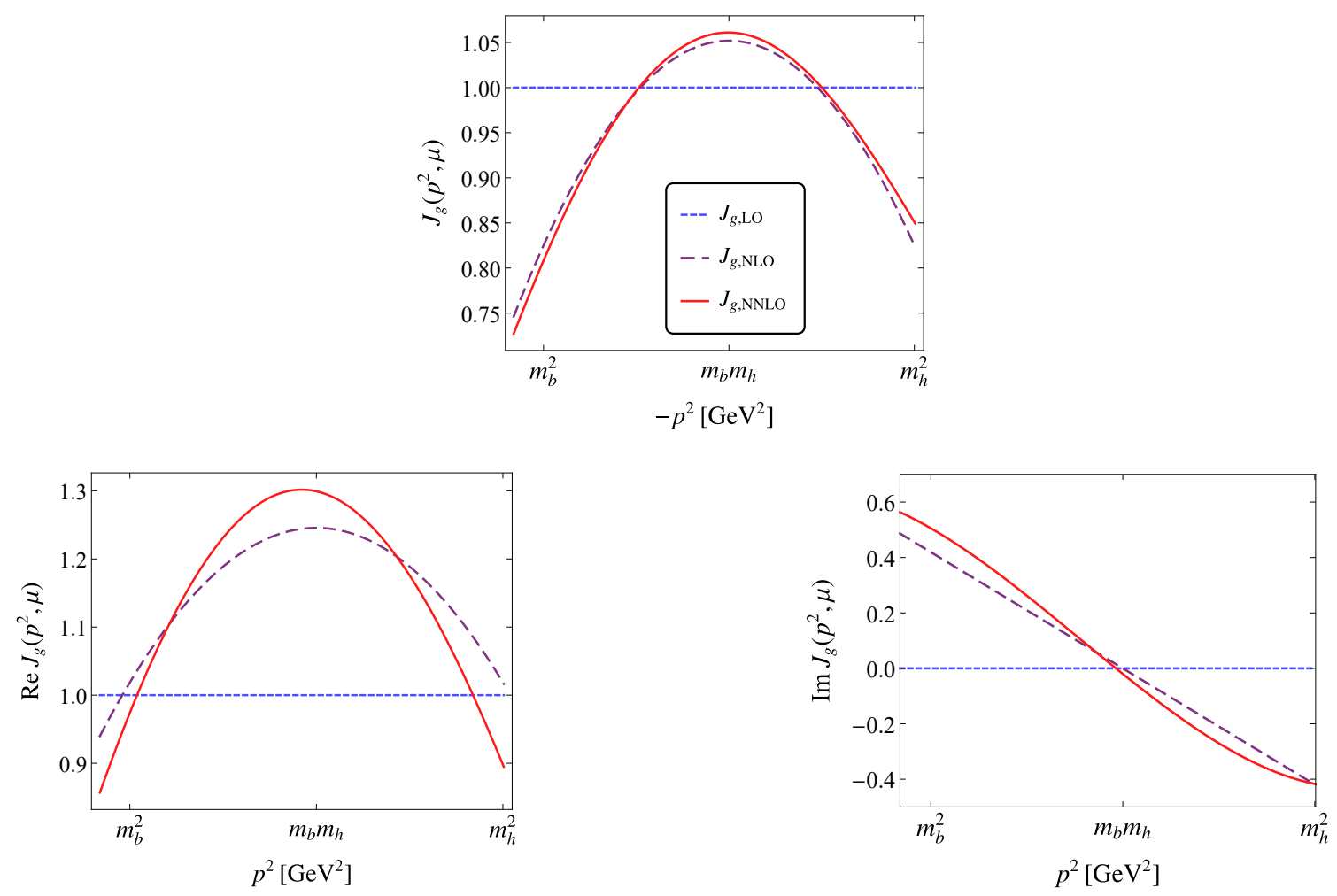

Figure 3. Renormalized jet function $J_{g}\left(p^{2}, \mu\right)$ in the space-like region $p^{2}<0$ (top) and in the time-like region $p^{2}>0$ (bottom), evaluated at tree level (dotted) and at one-loop (dashed) and two-loop order (solid) in perturbation theory. The renormalization scale is fixed at $\mu=\sqrt{m_{b} m_{h}}$. We use a logarithmic scale on the horizontal axis.

where

$$
\begin{aligned}
& \bar{K}_{F F}=\frac{L_{p}^{4}}{2}-\left(1+\frac{\pi^{2}}{6}\right) L_{p}^{2}+\left(\pi^{2}+4 \zeta_{3}\right) L_{p}+\frac{3}{2}-\frac{\pi^{2}}{3}-39 \zeta_{3}+\frac{119 \pi^{4}}{360} \\
& \bar{K}_{F A}=-L_{p}^{4}-\frac{11 L_{p}^{3}}{9}+\frac{85 L_{p}^{2}}{9}-\left(\frac{305}{27}+\frac{\pi^{2}}{2}+4 \zeta_{3}\right) L_{p}-\frac{317}{162}-\frac{65 \pi^{2}}{54}+\frac{793 \zeta_{3}}{18}-\frac{143 \pi^{4}}{360} \\
& \bar{K}_{A A}=\frac{L_{p}^{4}}{2}+\frac{11 L_{p}^{3}}{9}-\left(\frac{76}{9}-\frac{\pi^{2}}{6}\right) L_{p}^{2}+\left(\frac{296}{27}-\frac{11 \pi^{2}}{18}\right) L_{p}+\frac{154}{81}+\frac{85 \pi^{2}}{54}-\frac{49 \zeta_{3}}{18}+\frac{\pi^{4}}{15} \\
& \bar{K}_{F n_{f}}=\frac{4 L_{p}^{3}}{9}-\frac{20 L_{p}^{2}}{9}+\frac{76 L_{p}}{27}+\frac{14}{81}+\frac{5 \pi^{2}}{27}+\frac{8 \zeta_{3}}{9}, \\
& \bar{K}_{A n_{f}}=-\frac{4 L_{p}^{3}}{9}+\frac{20 L_{p}^{2}}{9}-\left(\frac{58}{27}-\frac{2 \pi^{2}}{9}\right) L_{p}-\frac{275}{81}-\frac{10 \pi^{2}}{27}-\frac{50 \zeta_{3}}{9}
\end{aligned}
$$

Expression (3.13) is the main result of this paper.

In figure 3 we show the renormalized jet function $J_{g}\left(p^{2}, \mu\right)$ as a function of $p^{2}$, for both space-like and time-like momenta. For $p^{2}>0$ the jet function has a non-zero imaginary part, which is shown in the lower-right plot. In the discussion of the Higgs-boson production amplitude $g g \rightarrow h$ via $b$-quark loops, the jet function is evaluated for $p^{2}$ values (of either 
sign) ranging between $m_{b}^{2}$ and $m_{h}^{2}$ [18], and this is therefore the range covered in the plots. The renormalization scale is fixed at the geometric mean $\mu=\sqrt{m_{b} m_{h}}$. We observe a good convergence of the perturbative expansion, as indicated by the fact that the two-loop contribution to the jet function is considerable smaller than the one-loop contribution for most values of $p^{2}$. Note that near the boundaries of the plots the logarithm $L_{p}=\ln \left(-p^{2} / \mu^{2}\right)$ takes parametrically large values of order $\ln \left(m_{h} / m_{b}\right)$. In order to obtain reliable results in these regions, these logarithms should be resummed to all orders of perturbation theory, as we will now discuss.

\section{RG evolution of the jet function}

For practical applications in the context of QCD factorization theorems, it is often necessary to evolve a jet function to a scale $\mu$ that is parametrically different from its "natural" scale $\mu_{j} \sim \sqrt{\left|p^{2}\right|}$. To this end, one needs to solve the RG evolution equation (3.2), which can be accomplished by transforming this equation to Laplace space $[14,32,39]$. Here we briefly discuss the solution at leading order in RG-improved perturbation theory, which can be obtained by starting from the solution for the jet function with an external photon derived in [12] and accounting for the different color factors. We find

$$
\begin{aligned}
J_{g}^{\mathrm{RGi}, \mathrm{LO}}\left(p^{2}, \mu\right)= & e^{-2 S_{g}\left(\mu_{j}, \mu\right)} \partial_{g}^{\mathrm{LO}}\left(\partial_{\eta}, \mu_{j}\right)\left(\frac{-p^{2}-i 0}{\mu_{j}^{2}}\right)^{a_{\Gamma}\left(\mu_{j}, \mu\right)+\eta} \\
& \times\left.\left[e^{-2 \gamma_{E} a_{\Gamma}\left(\mu_{j}, \mu\right)} \frac{\Gamma\left(1-a_{\Gamma}\left(\mu_{j}, \mu\right)-\eta\right) \Gamma(1+\eta)}{\Gamma\left(1+a_{\Gamma}\left(\mu_{j}, \mu\right)+\eta\right) \Gamma(1-\eta)}\right]^{\rho_{g}}\right|_{\eta=0},
\end{aligned}
$$

where both the Sudakov exponent

$$
S_{g}\left(\mu_{j}, \mu\right)=\frac{\Delta \Gamma_{0}}{4 \beta_{0}^{2}}\left[\frac{4 \pi}{\alpha_{s}\left(\mu_{j}\right)}\left(1-\frac{1}{r}-\ln r\right)+\left(\frac{\Delta \Gamma_{1}}{\Delta \Gamma_{0}}-\frac{\beta_{1}}{\beta_{0}}\right)(1-r+\ln r)+\frac{\beta_{1}}{2 \beta_{0}} \ln ^{2} r\right],
$$

with $r=\alpha_{s}(\mu) / \alpha_{s}\left(\mu_{j}\right)$, and the function

$$
a_{\Gamma}\left(\mu_{j}, \mu\right)=\frac{\Delta \Gamma_{0}}{2 \beta_{0}} \ln \frac{\alpha_{s}(\mu)}{\alpha_{s}\left(\mu_{j}\right)}
$$

differ from the corresponding objects in the photon case by the overall color factor $\left(C_{F}-C_{A}\right)$ (instead of $C_{F}$ ). Another important difference is the appearance of the exponent

$$
\rho_{g}=\frac{C_{F}-\frac{1}{2} C_{A}}{C_{F}-C_{A}}=\frac{1}{N_{c}^{2}+1},
$$

which strongly suppresses the deviation of the term shown in the second line of (4.1) from 1. Note that a consistent evaluation of the Sudakov exponent requires the two-loop coefficients of the cusp anomalous dimension and the QCD $\beta$-function.

The function $\mathcal{J}_{g}^{\mathrm{LO}}\left(\partial_{\eta}, \mu_{j}\right)$ in the solution (4.1) is a differential operator acting on functions of the auxiliary parameter $\eta$. It is defined by the identification $J_{g}\left(p^{2}, \mu_{j}\right) \equiv \mathcal{J}_{g}\left(L_{p}, \mu_{j}\right)$. 

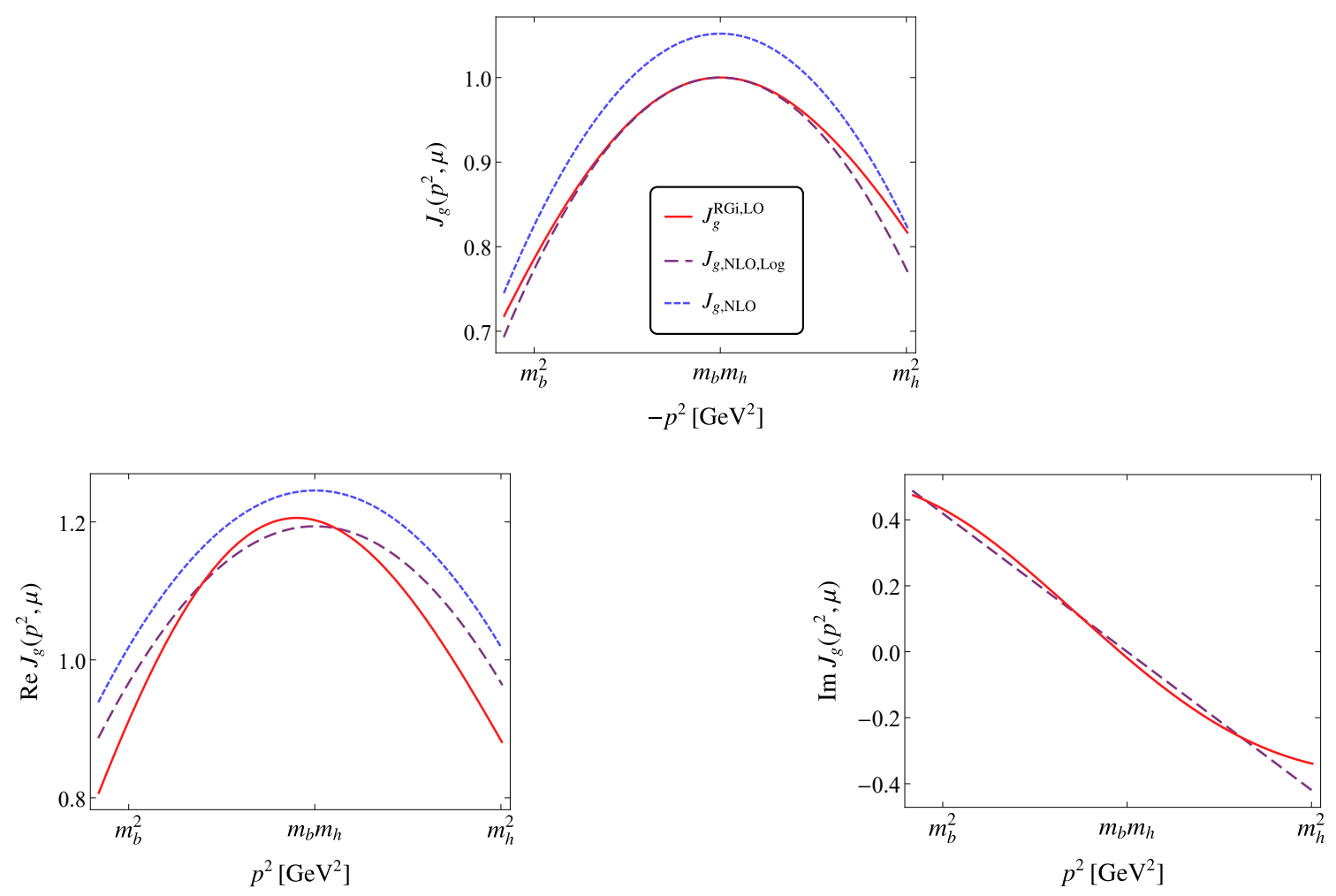

Figure 4. Renormalized jet function $J_{g}\left(p^{2}, \mu\right)$ in the space-like region $p^{2}<0$ (top) and in the time-like region $p^{2}>0$ (bottom), at leading order in RG-improved perturbation theory (solid lines). The renormalization scale is fixed to $\mu=\sqrt{m_{b} m_{h}}$, while the matching scale $\mu_{j}$ is chosen as $\mu_{j}^{2}=-p^{2}-i 0$. For comparison we also show the one-loop expression for the jet function (dotted lines) and the logarithmic terms in the one-loop expression (dashed lines). In the lower right plot these two curves overlap. We use a logarithmic scale on the horizontal axis.

At the matching scale $\mu_{j}$ chosen as $\mu_{j}^{2} \sim p^{2}$, the expression for the jet function in (3.13) is free of large logarithms. In fact, at leading order in RG-improved perturbation theory one has $\operatorname{Jg}_{g}^{\mathrm{LO}}\left(L_{p}, \mu_{j}\right)=1$. Using this initial condition, we find the simple result

$$
J_{g}^{\mathrm{RGi}, \mathrm{LO}}\left(p^{2}, \mu\right)=e^{-2 S_{g}\left(\mu_{j}, \mu\right)}\left(\frac{-p^{2}-i 0}{\mu_{j}^{2}}\right)^{a_{\Gamma}\left(\mu_{j}, \mu\right)}\left[e^{-2 \gamma_{E} a_{\Gamma}\left(\mu_{j}, \mu\right)} \frac{\Gamma\left(1-a_{\Gamma}\left(\mu_{j}, \mu\right)\right)}{\Gamma\left(1+a_{\Gamma}\left(\mu_{j}, \mu\right)\right)}\right]^{\rho_{g}} .
$$

This formula resums the leading logarithmic corrections to the jet function to all orders of perturbation theory.

In figure 4 we show the resummed jet function $J_{g}\left(p^{2}, \mu\right)$ at leading-order in RGimproved perturbation theory (solid lines), for both space-like and time-like momentum transfer. As in figure 3 we fix the renormalization scale at $\mu=\sqrt{m_{b} m_{h}}$. The matching scale $\mu_{j}$ is chosen as $\mu_{j}^{2}=-p^{2}$ so as to eliminate large logarithms from the matching condition. For comparison, the dotted lines show the one-loop result for the jet function in fixed-order perturbation theory, while the dashed lines show the contribution of only the logarithmic terms in the one-loop result. Comparing the solid and dashed curves, we see that the 
resummation of higher-order logarithmic effects is important if $\left|p^{2}\right|$ is much larger or much smaller than $\mu^{2}=m_{b} m_{h}$, which is indeed what one would expect. Comparing the dotted and dashed curves shows that the non-logarithmic contributions in the jet function are however also significant. In order to derive a more accurate result at next-to-leading order in RG-improved perturbation theory, it would be necessary to calculate the two-loop contribution to the anomalous dimension in (3.3). This is an interesting target for future research.

\section{Conclusions}

We have presented a detailed study of the quark jet function with an external gluon, $J_{g}\left(p^{2}, \mu\right)$ in $(1.4)$, which plays a central role in the factorization theorem for the lightquark induced contribution to the $g g \rightarrow h$ production amplitude of the Higgs boson at hadron colliders. We have calculated this jet function up to two-loop order, derived its one-loop anomalous dimension, and solved its RG evolution equation at leading order in RG-improved perturbation theory. We find that the quark jet function with a radiated gluon exhibits a more interesting color structure than the corresponding jet function with a radiated photon, considered in [12]. While the one-loop gluon jet function can be obtained from the photon jet function by the simple replacement $C_{F} \rightarrow\left(C_{F}-C_{A}\right)$ of color factors, the one-loop anomalous dimension of the gluon jet function shown in (3.3) features two different color factors, $\left(C_{F}-C_{A}\right)$ and $\left(C_{F}-\frac{C_{A}}{2}\right)$. Consequently, the color factor of the Lange-Neubert kernel is no longer the same as that of the Sudakov term, and as a result the leading-order solution to the RG evolution equation in (4.1) involves the non-trivial exponent $\rho_{g}=\frac{1}{10}$. Starting at two-loop order, there is no simple relation between the two-loop coefficients of the gluon and photon jet functions.

The results derived in this paper provide an important input to the factorization theorem for the already-mentioned gluon-gluon fusion process $g g \rightarrow h$. The quark jet function with an external gluon is a crucial ingredient in two $d$-dimensional refactorization conditions, which allow us to remove the endpoint divergences in the factorization theorem using suitable subtractions $[8,10,11]$. Moreover, the knowledge of the RG equation of the jet function will enable us to derive some logarithmically enhanced contributions to the three-loop $g g \rightarrow h$ amplitude, which may be relevant for phenomenology [18].

\section{Acknowledgments}

We are grateful to Thomas Becher for useful discussions. The research of M.N., M.S. and X.W. is supported by the Cluster of Excellence Precision Physics, Fundamental Interactions and Structure of Matter (PRISMA ${ }^{+}$, EXC 2118/1) within the German Excellence Strategy (project ID 39083149). The research of Z.L.L. is supported by the Swiss National Science Foundation under grant 200020_182038.

\section{A Master integrals}

After integration-by-parts reduction, the remaining master integrals have vanishing exponents $a_{3}=a_{8}=a_{12}=a_{13}=a_{14}=a_{15}=0$. They can be expressed in terms of the integral 
family

$$
\begin{aligned}
& \int d^{d} l_{1} \int d^{d} l_{2} \frac{1}{\mathcal{D}_{1}^{a_{1}} \mathcal{D}_{2}^{a_{2}} \mathcal{D}_{4}^{a_{4}} \mathcal{D}_{5}^{a_{5}} \mathcal{D}_{6}^{a_{6}} \mathcal{D}_{7}^{a_{7}} \mathcal{D}_{9}^{a_{9}} \mathcal{D}_{10}^{a_{10}} \mathcal{D}_{11}^{a_{11}}} \\
& =-\pi^{d} e^{-2 \epsilon \gamma_{E}}\left(-p^{2}-i 0\right)^{d-\sum_{i=1}^{9} a_{i}}(\bar{n} \cdot p)^{-a_{10}-a_{11}} G_{a_{1}, a_{2}, a_{4}, a_{5}, a_{6}, a_{7}, a_{9}, a_{10}, a_{11}},
\end{aligned}
$$

where the propagators $\mathcal{D}_{i}$ have been defined in (2.5). Two of the ten master integrals can be evaluated in closed form, yielding

$$
\begin{aligned}
& G_{1,1,0,0,1,0,0,0,0}=e^{2 \epsilon \gamma_{E}} \frac{\Gamma^{3}(1-\epsilon) \Gamma(2 \epsilon-1)}{\Gamma(3-3 \epsilon)}, \\
& G_{1,1,1,1,0,0,0,0,0}=e^{2 \epsilon \gamma_{E}} \frac{\Gamma^{4}(1-\epsilon) \Gamma^{2}(\epsilon)}{\Gamma^{2}(2-2 \epsilon)} .
\end{aligned}
$$

For the remaining eight master integrals we quote the Laurent expansions in $\epsilon$ up to $\zeta$-values of weight 6 , finding

$$
\begin{aligned}
G_{0,1,0,1,1,1,1,1,0}= & \frac{1}{2 \epsilon^{4}}-\frac{\pi^{2}}{2 \epsilon^{2}}-\frac{83 \zeta_{3}}{6 \epsilon}-\frac{59 \pi^{4}}{240}+\left(\frac{79 \pi^{2} \zeta_{3}}{12}-\frac{587 \zeta_{5}}{10}\right) \epsilon+\left(\frac{2567 \zeta_{3}^{2}}{18}+\frac{59 \pi^{6}}{3024}\right) \epsilon^{2} \\
& +\mathcal{O}\left(\epsilon^{3}\right), \\
G_{1,1,0,1,1,0,1,1,0}= & -\frac{1}{2 \epsilon^{4}}+\frac{\pi^{2}}{2 \epsilon^{2}}+\frac{83 \zeta_{3}}{6 \epsilon}+\frac{59 \pi^{4}}{240}+\left(\frac{587 \zeta_{5}}{10}-\frac{79 \pi^{2} \zeta_{3}}{12}\right) \epsilon+\left(-\frac{2567 \zeta_{3}^{2}}{18}-\frac{59 \pi^{6}}{3024}\right) \epsilon^{2} \\
& +\mathcal{O}\left(\epsilon^{3}\right), \\
G_{1,1,1,1,1,1,0,0,1}= & \frac{1}{\epsilon^{4}}+\frac{\pi^{2}}{4 \epsilon^{2}}+\frac{23 \zeta_{3}}{6 \epsilon}-\frac{11 \pi^{4}}{360}+\left(-\frac{13 \pi^{2} \zeta_{3}}{4}-\frac{309 \zeta_{5}}{10}\right) \epsilon+\left(-\frac{1661 \zeta_{3}^{2}}{18}-\frac{2897 \pi^{6}}{18144}\right) \epsilon^{2} \\
& +\mathcal{O}\left(\epsilon^{3}\right), \\
G_{1,1,0,1,1,1,1,0,0}= & \frac{1}{\epsilon^{4}}-\frac{\pi^{2}}{\epsilon^{2}}-\frac{83 \zeta_{3}}{3 \epsilon}-\frac{59 \pi^{4}}{120}+\left(\frac{79 \pi^{2} \zeta_{3}}{6}-\frac{587 \zeta_{5}}{5}\right) \epsilon+\left(\frac{2567 \zeta_{3}^{2}}{9}+\frac{59 \pi^{6}}{1512}\right) \epsilon^{2} \\
& +\mathcal{O}\left(\epsilon^{3}\right), \\
G_{0,1,1,1,1,1,0,1,1}= & \frac{1}{\epsilon^{4}}+\frac{7 \pi^{2}}{12 \epsilon^{2}}-\frac{19 \zeta_{3}}{6 \epsilon}+\frac{\pi^{4}}{30}+\left(-\frac{149 \pi^{2} \zeta_{3}}{36}-\frac{399 \zeta_{5}}{10}\right) \epsilon+\left(-\frac{199 \zeta_{3}^{2}}{9}-\frac{159 \pi^{6}}{1120}\right) \epsilon^{2} \\
& +\mathcal{O}\left(\epsilon^{3}\right), \\
& +\frac{1}{2 \epsilon^{2}}+\frac{5}{2 \epsilon}+\left(\frac{19}{2}+\frac{\pi^{2}}{12}\right)+\left(\frac{65}{2}+\frac{5 \pi^{2}}{12}-\frac{13 \zeta_{3}}{3}\right) \epsilon \\
& +\left(\frac{211}{2}+\frac{19 \pi^{2}}{12}-\frac{65 \zeta_{3}}{3}-\frac{41 \pi^{4}}{720}\right) \epsilon^{2} \\
& +\left(\frac{665}{2}+\frac{65 \pi^{2}}{12}-\frac{247 \zeta_{3}}{3}-\frac{41 \pi^{4}}{144}-\frac{13 \pi^{2} \zeta_{3}}{18}-\frac{121 \zeta_{5}}{5}\right) \epsilon^{3} \\
G_{0,1,0,1,1,1,0,0,0} & \left(\frac{2059}{2}+\frac{211 \pi^{2}}{12}-\frac{845 \zeta_{3}}{3}-\frac{779 \pi^{4}}{720}-\frac{65 \pi^{2} \zeta_{3}}{18}-121 \zeta_{5}+\frac{169 \zeta_{3}^{2}}{9}-\frac{127 \pi^{6}}{2016}\right) \epsilon^{4} \\
& (\mathrm{~A} .8
\end{aligned}
$$




$$
\begin{aligned}
G_{1,0,1,1,1,0,0,0,1}= & \frac{\pi^{2}}{12 \epsilon^{2}}+\frac{7 \zeta_{3}}{2 \epsilon}+\frac{11 \pi^{4}}{72}+\left(\frac{93 \zeta_{5}}{2}-\frac{29 \pi^{2} \zeta_{3}}{36}\right) \epsilon+\left(\frac{433 \pi^{6}}{3360}-\frac{70 \zeta_{3}^{2}}{3}\right) \epsilon^{2} \\
& +\mathcal{O}\left(\epsilon^{3}\right) \\
G_{1,1,1,1,1,0,0,1,1}= & \frac{\pi^{2}}{3 \epsilon^{2}}-\frac{7 \zeta_{3}}{\epsilon}+\frac{23 \pi^{4}}{360}+\left(-\frac{8 \pi^{2} \zeta_{3}}{9}-9 \zeta_{5}\right) \epsilon+\left(\frac{421 \zeta_{3}^{2}}{6}+\frac{803 \pi^{6}}{45360}\right) \epsilon^{2} \\
& +\mathcal{O}\left(\epsilon^{3}\right) .
\end{aligned}
$$

\section{B Bare two-loop coefficients to $\mathcal{O}\left(\epsilon^{2}\right)$}

In (2.6) and (2.7), the expansion coefficients in the two-loop expression for the bare jet function (2.1) have been shown up to terms of $\mathcal{O}\left(\epsilon^{0}\right)$ in the Laurent series. However, we have also obtained expressions for these coefficients to higher order in $\epsilon$. If in the future one aims at a calculation of the renormalized jet function at three-loop order, then the coefficients are needed up to terms of $\mathcal{O}\left(\epsilon^{2}\right)$. We find

$$
\begin{aligned}
& K_{F F}=\frac{2}{\epsilon^{4}}+\frac{1}{\epsilon^{2}}\left(-2-\frac{\pi^{2}}{3}\right)+\frac{1}{\epsilon}\left(-4-\frac{\pi^{2}}{2}-\frac{46 \zeta_{3}}{3}\right)+\left(-\frac{13}{2}-\frac{\pi^{2}}{6}-39 \zeta_{3}+\frac{\pi^{4}}{5}\right) \\
& +\left(-\frac{33}{4}+\frac{2 \pi^{2}}{3}-\frac{431 \zeta_{3}}{3}-\frac{11 \pi^{4}}{15}+\frac{26 \pi^{2} \zeta_{3}}{9}+\frac{766 \zeta_{5}}{5}\right) \epsilon \\
& +\left(-\frac{19}{8}+\frac{37 \pi^{2}}{12}-\frac{1780 \zeta_{3}}{3}-\frac{116 \pi^{4}}{45}+\frac{89 \pi^{2} \zeta_{3}}{6}-153 \zeta_{5}+\frac{3707 \pi^{6}}{5670}+\frac{2077 \zeta_{3}^{2}}{9}\right) \epsilon^{2} \\
& +O\left(\epsilon^{3}\right) \text {, } \\
& K_{F A}=-\frac{4}{\epsilon^{4}}+\frac{11}{6 \epsilon^{3}}+\frac{1}{\epsilon^{2}}\left(\frac{139}{18}+\frac{\pi^{2}}{2}\right)+\frac{1}{\epsilon}\left(\frac{319}{27}-\frac{\pi^{2}}{18}+\frac{80 \zeta_{3}}{3}\right) \\
& +\left(\frac{1087}{162}-\frac{83 \pi^{2}}{54}+\frac{485 \zeta_{3}}{18}-\frac{49 \pi^{4}}{360}\right) \\
& +\left(-\frac{42551}{972}-\frac{589 \pi^{2}}{162}+\frac{7945 \zeta_{3}}{54}+\frac{229 \pi^{4}}{720}-\frac{9 \pi^{2} \zeta_{3}}{2}-\frac{762 \zeta_{5}}{5}\right) \epsilon \\
& +\left(-\frac{1581791}{5832}-\frac{6919 \pi^{2}}{972}+\frac{65527 \zeta_{3}}{81}+\frac{4433 \pi^{4}}{2160}-\frac{1529 \pi^{2} \zeta_{3}}{108}-\frac{427 \zeta_{5}}{30}\right. \\
& \left.-\frac{17471 \pi^{6}}{22680}-\frac{5659 \zeta_{3}^{2}}{18}\right) \epsilon^{2}+O\left(\epsilon^{3}\right), \\
& K_{A A}=\frac{2}{\epsilon^{4}}-\frac{11}{6 \epsilon^{3}}+\frac{1}{\epsilon^{2}}\left(-\frac{103}{18}-\frac{\pi^{2}}{6}\right)+\frac{1}{\epsilon}\left(-\frac{413}{54}+\frac{11 \pi^{2}}{18}-\frac{34 \zeta_{3}}{3}\right) \\
& +\left(\frac{100}{81}+\frac{47 \pi^{2}}{27}+\frac{259 \zeta_{3}}{18}-\frac{23 \pi^{4}}{360}\right) \\
& +\left(\frac{14375}{243}+\frac{979 \pi^{2}}{324}-\frac{103 \zeta_{3}}{54}+\frac{1117 \pi^{4}}{2160}+\frac{29 \pi^{2} \zeta_{3}}{18}-\frac{4 \zeta_{5}}{5}\right) \epsilon \\
& +\left(\frac{220477}{729}+\frac{935 \pi^{2}}{243}-\frac{17257 \zeta_{3}}{81}+\frac{769 \pi^{4}}{1296}-\frac{131 \pi^{2} \zeta_{3}}{108}+\frac{5947 \zeta_{5}}{30}+\frac{881 \pi^{6}}{7560}+\frac{1505 \zeta_{3}^{2}}{18}\right) \epsilon^{2} \\
& +O\left(\epsilon^{3}\right) \text {, }
\end{aligned}
$$




$$
\begin{aligned}
K_{F n_{f}=}= & \frac{2}{3 \epsilon^{3}}-\frac{10}{9 \epsilon^{2}}+\frac{1}{\epsilon}\left(-\frac{20}{27}+\frac{\pi^{2}}{9}\right)+\left(\frac{230}{81}+\frac{5 \pi^{2}}{27}+\frac{64 \zeta_{3}}{9}\right) \\
& +\left(\frac{4591}{243}+\frac{10 \pi^{2}}{81}+\frac{320 \zeta_{3}}{27}+\frac{19 \pi^{4}}{180}\right) \epsilon \\
& +\left(\frac{113863}{1458}-\frac{115 \pi^{2}}{243}+\frac{640 \zeta_{3}}{81}+\frac{19 \pi^{4}}{108}-\frac{32 \pi^{2} \zeta_{3}}{27}+\frac{544 \zeta_{5}}{15}\right) \epsilon^{2}+O\left(\epsilon^{3}\right), \\
K_{A n_{f}=} & \frac{2}{3 \epsilon^{3}}+\frac{10}{9 \epsilon^{2}}+\frac{1}{\epsilon}\left(\frac{11}{27}-\frac{2 \pi^{2}}{9}\right)+\left(-\frac{491}{81}-\frac{10 \pi^{2}}{27}-\frac{106 \zeta_{3}}{9}\right) \\
& +\left(-\frac{8839}{243}-\frac{67 \pi^{2}}{162}-\frac{530 \zeta_{3}}{27}-\frac{167 \pi^{4}}{540}\right) \epsilon \\
& +\left(-\frac{111719}{729}+\frac{271 \pi^{2}}{486}-\frac{1690 \zeta_{3}}{81}-\frac{167 \pi^{4}}{324}+\frac{61 \pi^{2} \zeta_{3}}{27}-\frac{1474 \zeta_{5}}{15}\right) \epsilon^{2}+O\left(\epsilon^{3}\right) .
\end{aligned}
$$

Open Access. This article is distributed under the terms of the Creative Commons Attribution License (CC-BY 4.0), which permits any use, distribution and reproduction in any medium, provided the original author(s) and source are credited.

\section{References}

[1] C.W. Bauer, S. Fleming, D. Pirjol, I.Z. Rothstein and I.W. Stewart, Hard scattering factorization from effective field theory, Phys. Rev. D 66 (2002) 014017 [hep-ph/0202088] [INSPIRE].

[2] M. Beneke, A.P. Chapovsky, M. Diehl and T. Feldmann, Soft collinear effective theory and heavy to light currents beyond leading power, Nucl. Phys. B 643 (2002) 431 [hep-ph/0206152] [INSPIRE].

[3] I. Moult, I.W. Stewart and G. Vita, Subleading power factorization with radiative functions, JHEP 11 (2019) 153 [arXiv:1905.07411] [INSPIRE].

[4] M. Beneke, M. Garny, R. Szafron and J. Wang, Violation of the Kluberg-Stern-Zuber theorem in SCET, JHEP 09 (2019) 101 [arXiv: 1907.05463] [INSPIRE].

[5] I. Moult, I.W. Stewart, G. Vita and H.X. Zhu, The soft quark Sudakov, JHEP 05 (2020) 089 [arXiv: 1910.14038] [INSPIRE].

[6] M. Beneke, A. Broggio, S. Jaskiewicz and L. Vernazza, Threshold factorization of the Drell-Yan process at next-to-leading power, JHEP 07 (2020) 078 [arXiv:1912.01585] [INSPIRE].

[7] I. Moult, G. Vita and K. Yan, Subleading power resummation of rapidity logarithms: the energy-energy correlator in $N=4$ SYM, JHEP 07 (2020) 005 [arXiv:1912.02188] [INSPIRE].

[8] Z.L. Liu and M. Neubert, Factorization at subleading power and endpoint-divergent convolutions in $h \rightarrow \gamma \gamma$ decay, JHEP 04 (2020) 033 [arXiv: 1912.08818] [INSPIRE].

[9] M. Beneke, M. Garny, S. Jaskiewicz, R. Szafron, L. Vernazza and J. Wang, Large- $x$ resummation of off-diagonal deep-inelastic parton scattering from d-dimensional refactorization, JHEP 10 (2020) 196 [arXiv: 2008. 04943] [INSPIRE]. 
[10] Z.L. Liu, B. Mecaj, M. Neubert and X. Wang, Factorization at subleading power, Sudakov resummation, and endpoint divergences in soft-collinear effective theory, Phys. Rev. D 104 (2021) 014004 [arXiv:2009.04456] [INSPIRE].

[11] Z.L. Liu, B. Mecaj, M. Neubert and X. Wang, Factorization at subleading power and endpoint divergences in $h \rightarrow \gamma \gamma$ decay. Part II. Renormalization and scale evolution, JHEP 01 (2021) 077 [arXiv: 2009.06779] [INSPIRE].

[12] Z.L. Liu and M. Neubert, Two-loop radiative jet function for exclusive B-meson and Higgs decays, JHEP 06 (2020) 060 [arXiv:2003.03393] [INSPIRE].

[13] E. Lunghi, D. Pirjol and D. Wyler, Factorization in leptonic radiative $B \rightarrow$ rev decays, Nucl. Phys. B 649 (2003) 349 [hep-ph/0210091] [inSPIRE].

[14] S.W. Bosch, R.J. Hill, B.O. Lange and M. Neubert, Factorization and Sudakov resummation in leptonic radiative B decay, Phys. Rev. D 67 (2003) 094014 [hep-ph/0301123] [INSPIRE].

[15] V. Del Duca, High-energy Bremsstrahlung theorems for soft photons, Nucl. Phys. B 345 (1990) 369 [INSPIRE].

[16] D. Bonocore, E. Laenen, L. Magnea, S. Melville, L. Vernazza and C.D. White, $A$ factorization approach to next-to-leading-power threshold logarithms, JHEP 06 (2015) 008 [arXiv: 1503.05156] [INSPIRE].

[17] D. Bonocore, E. Laenen, L. Magnea, L. Vernazza and C.D. White, Non-Abelian factorisation for next-to-leading-power threshold logarithms, JHEP 12 (2016) 121 [arXiv:1610.06842] [INSPIRE].

[18] Z. Liu, M. Neubert, M. Schnubel and X. Wang, Renormalization of the radiative gluon jet function, in preparation.

[19] R.J. Hill and M. Neubert, Spectator interactions in soft collinear effective theory, Nucl. Phys. B 657 (2003) 229 [hep-ph/0211018] [INSPIRE].

[20] A.V. Manohar, T. Mehen, D. Pirjol and I.W. Stewart, Reparameterization invariance for collinear operators, Phys. Lett. B 539 (2002) 59 [hep-ph/0204229] [INSPIRE].

[21] G. 't Hooft, The background field method in gauge field theories, in Procs. $12^{\text {th }}$ annual winter school of theoretical physics, Karpacz, Poland (1975) [INSPIRE].

[22] D.G. Boulware, Gauge dependence of the effective action, Phys. Rev. D 23 (1981) 389 [INSPIRE].

[23] L.F. Abbott, The background field method beyond one loop, Nucl. Phys. B 185 (1981) 189 [INSPIRE].

[24] L.F. Abbott, Introduction to the background field method, Acta Phys. Polon. B 13 (1982) 33 [INSPIRE].

[25] K.A. Meissner, The background field method in the axial gauge, Acta Phys. Polon. B 17 (1986) 409 [INSPIRE].

[26] T. Becher and G. Bell, The gluon jet function at two-loop order, Phys. Lett. B 695 (2011) 252 [arXiv: 1008.1936] [INSPIRE].

[27] R.N. Lee, LiteRed 1.4: a powerful tool for reduction of multiloop integrals, J. Phys. Conf. Ser. 523 (2014) 012059 [arXiv: 1310.1145] [INSPIRE].

[28] A.V. Smirnov and F.S. Chuharev, FIRE6: Feynman Integral REduction with modular arithmetic, Comput. Phys. Commun. 247 (2020) 106877 [arXiv:1901.07808] [INSPIRE]. 
[29] J. Klappert, F. Lange, P. Maierhöfer and J. Usovitsch, Integral reduction with Kira 2.0 and finite field methods, Comput. Phys. Commun. 266 (2021) 108024 [arXiv:2008.06494] [INSPIRE].

[30] S.W. Bosch, B.O. Lange, M. Neubert and G. Paz, Factorization and shape function effects in inclusive B meson decays, Nucl. Phys. B 699 (2004) 335 [hep-ph/0402094] [InSPIRE].

[31] A.G. Grozin and M. Neubert, Asymptotics of heavy meson form-factors, Phys. Rev. D 55 (1997) 272 [hep-ph/9607366] [INSPIRE].

[32] B.O. Lange and M. Neubert, Renormalization group evolution of the $B$ meson light cone distribution amplitude, Phys. Rev. Lett. 91 (2003) 102001 [hep-ph/0303082] [INSPIRE].

[33] Z.L. Liu, B. Mecaj, M. Neubert, X. Wang and S. Fleming, Renormalization and scale evolution of the soft-quark soft function, JHEP 07 (2020) 104 [arXiv: 2005. 03013] [INSPIRE].

[34] G.T. Bodwin, J.-H. Ee, J. Lee and X.-P. Wang, Analyticity, renormalization, and evolution of the soft-quark function, Phys. Rev. D 104 (2021) 016010 [arXiv:2101.04872] [InSPIRE].

[35] G.T. Bodwin, J.-H. Ee, J. Lee and X.-P. Wang, Renormalization of the radiative jet function, Phys. Rev. D 104 (2021) 116025 [arXiv:2107.07941] [InSPIRE].

[36] T. Becher and M. Neubert, On the structure of infrared singularities of gauge-theory amplitudes, JHEP 06 (2009) 081 [Erratum ibid. 11 (2013) 024] [arXiv:0903.1126] [INSPIRE].

[37] G.P. Korchemsky and A.V. Radyushkin, Renormalization of the Wilson loops beyond the leading order, Nucl. Phys. B 283 (1987) 342 [inSPIRE].

[38] I.A. Korchemskaya and G.P. Korchemsky, On lightlike Wilson loops, Phys. Lett. B 287 (1992) 169 [INSPIRE].

[39] A.M. Galda and M. Neubert, Evolution of the B-meson light-cone distribution amplitude in Laplace space, Phys. Rev. D 102 (2020) 071501 [arXiv:2006.05428] [INSPIRE]. 